\title{
Candida auris Infection and Biofilm Formation: Going Beyond the Surface
}

\author{
Mark V. Horton $^{1}$ • Jeniel E. Nett ${ }^{1}$ \\ Published online: 17 July 2020 \\ (C) The Author(s) 2020
}

\section{Abstract}

\section{Purpose of Review}

Emergent fungal pathogen $C$. auris is spreading in hospitals throughout the world and mortality rates for patients with invasive disease approach $60 \%$. This species exhibits a heightened capacity to colonize skin, persist on hospital surfaces, rapidly disseminate in healthcare settings, and resist antifungal therapy.

\section{Recent Findings}

Current investigations show that $C$. auris produces biofilms, surface-adherent communities that resist antifungals and withstand desiccation. These biofilms form when $C$. auris is growing on skin or in conditions expected in the hospital environment and on implanted medical devices.

\section{Summary}

Here, we will highlight the topic of biofilm formation by $C$. auris. We illustrate how this process influences resistance to antimicrobials and promotes nosocomial transmission.

Keywords Candida auris $\cdot$ Biofilm $\cdot$ Pathogenicity $\cdot$ Skin $\cdot$ Colonization $\cdot$ Antifungal resistance

\section{Introduction}

Candida auris was first described in 2009 , following the isolation of this new species from the ear canal of a patient in Japan [1]. Since its discovery, we have witnessed numerous outbreaks of $C$. auris in healthcare centers throughout the world [2]. C. auris represents the first fungal pathogen to be termed a global public health threat, which is based on its ability to spread patient-to-patient and cause invasive disease with high mortality [2-4]. Other obstacles in the treatment of C. auris include its profound resistance to antifungal drugs as well as delays in diagnosis and treatment, as this new pathogen is not present in many clinical diagnostic systems $[5,6]$.

This article is part of the Topical Collection on Mycology

Jeniel E. Nett

jenett@medicine.wisc.edu

1 Departments of Medicine and Medical Microbiology \& Immunology, University of Wisconsin-Madison, Madison, WI, USA
The rampant nosocomial transmission observed for C. auris is unique to this species of Candida. Recent investigations are just beginning to shed light on the $C$. auris traits that may be involved in hospital spread. Like other Candida

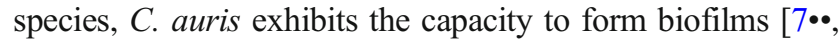
$8 \bullet, 9 \bullet, 11 \bullet \bullet$. Here, we highlight the characteristics of biofilms formed by $C$. auris and describe how this mode of growth contributes to the ability of $C$. auris to colonize skin, persist in the hospital environment, resist antimicrobial therapy, and cause invasive disease (Fig. 1).

\section{What Is the Clinical Presentation of $C$. auris Infection?}

C. auris infection occurs at a variety of clinical sites, including the bloodstream, wounds, and the urinary tract $[6,12,13]$. In addition, $C$. auris colonizes skin, nares, wounds, and urine, as a marker of disease risk [3, 6, 14]. Similar to patients with candidiasis caused by other species, patients with invasive 


\section{C. auris biofilm}

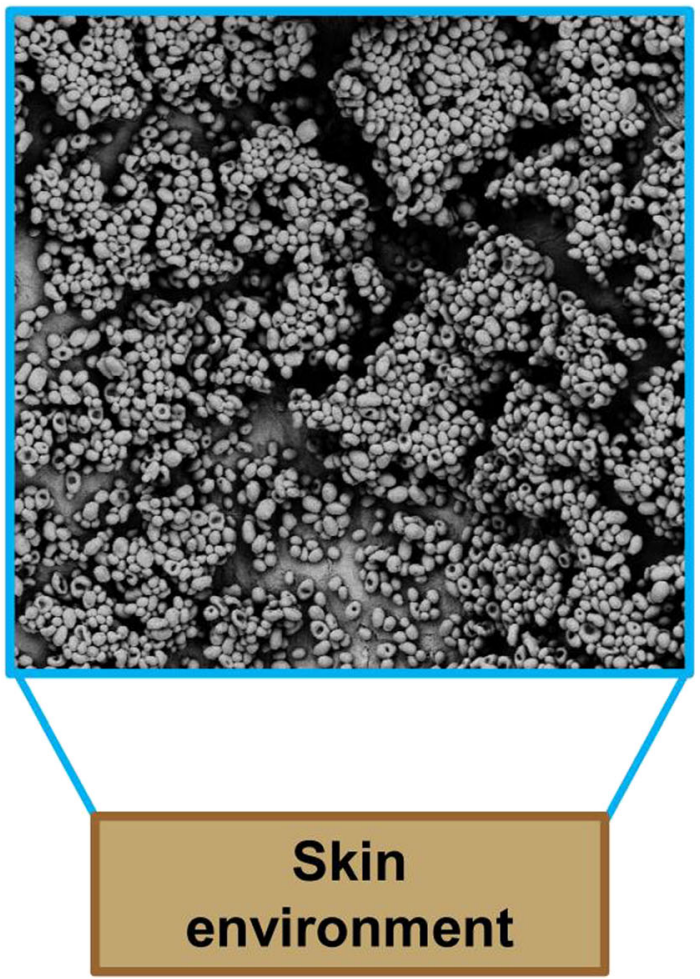

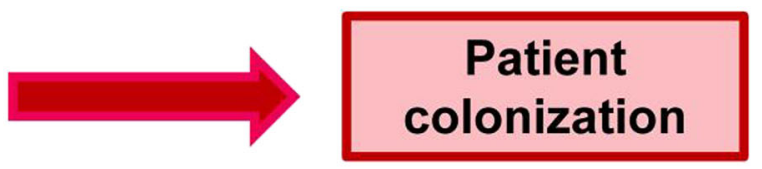
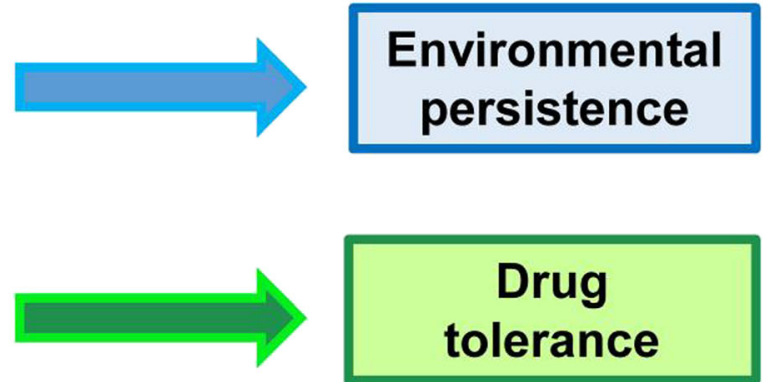

Fig. 1 C. auris forms high-density biofilms in skin niche conditions and in hospitalized settings. Scanning electron microscopy shows $C$. auris growing as a biofilm on porcine skin ex vivo. Dense biofilm formation likely contributes to $C$. auris pathogenicity and spread in healthcare settings

C. auris infection often present with fever or sepsis $[13,15$, 16]. Hospitalized patients and those residing in long-term care facilities are particularly at risk for C. auris infection [6]. Other specific risk factors associated with acquiring $C$. auris infection, as opposed to non-auris candidemia, include prolonged admission to an intensive care unit, prior antimicrobial therapy, central vascular catheter placement, total parenteral nutrition (TPN) administration, and the presence of underlying comorbidities, including respiratory, neurological, or kidney disease [3, 18-20, 22].

Patients that develop C. auris infection have frequently undergone numerous medical procedures, including the implantation of vascular catheters, urinary catheters, and percutaneous enteral feeding tubes $[6,16,18-20]$. The presence of central catheters in these patients is particularly high, with one study revealing indwelling lines in $>97 \%$ of patients [17]. Retrospective analyses have shown significantly higher use of central venous catheters in patients with $C$. auris infection compared to those with non-auris candidemia $[17,18]$. This suggests a role for vascular catheters in the pathogenesis of candidemia for $C$. auris. Indeed, catheters appear to be a more common source of infection for patients with C. auris $(89 \%)$ versus non-C. auris candidemia (46\%) [17]. Catheterassociated bloodstream infection involves the formation of biofilm on a catheter surface, which is followed by dissemination into the blood. Like other Candida spp., C. auris forms biofilms on artificial surfaces and this mode of growth is presumably involved in catheter colonization by C. auris $[2,7 \bullet \bullet, 8 \bullet, 9 \bullet, 10,11 \bullet, 12,20,21]$. In addition, C. auris has been implicated in other device-associated infections, including central nervous system infection in the setting of neurosurgical device placement and prosthetic joint infection $[22,23]$. Biofilm formation is similarly anticipated to be involved in C. auris infection involving these and other medical devices [21, 24].

\section{Why Is C. auris Spreading in Hospitals?}

Within healthcare settings, $C$. auris has demonstrated a propensity for rapid spread among patients [6, 12, 25]. Factors contributing to transmission include the organism's capacity to colonize skin and to persist in the hospital environment. For example, screening of patients during a $C$. auris epidemic revealed colonization for $11 \%$ of patients within the involved healthcare facilities [6]. Approximately $75 \%$ of patients were colonized in the axilla or groin, with the remaining $25 \%$ colonized in the nares only. Many of these patients remained consistently colonized, with $C$. auris colonization documented for close to 200 days [6]. In addition, reports describe the 
persistence of $C$. auris on skin despite daily cleansing with chlorhexidine $[3,26]$. The propensity of $C$. auris to colonize skin is concerning in light of the pathogen's ability to persist in the environment and on medical equipment. For instance, the investigation of an outbreak in the United Kingdom cultured $C$. auris from axillary thermometers and linked these reusable devices to the transmission of this pathogen in a neurosurgical critical care unit [25].

C. auris can also persist on various fomites and surfaces within the hospital setting. Common areas of isolation include curtains, floors, windows, bedrails, equipment monitors, and IV poles $[3,6]$. In vitro studies show that $C$. auris remains viable for up to 2 weeks under similar environmental conditions [10, 27]. This suggests that contaminated medical equipment and hospital surfaces may pose infectious risks for weeks. Further complicating control of $C$. auris transmission is the relative resistance of $C$. auris to disinfectants that are commonly used in hospitals, including quaternary ammonia compounds [28]. For cleaning of surfaces harboring C. auris, alternative disinfectants are currently recommended by the Centers for Disease Control and Prevention (https://www. cdc.gov/fungal/candida-auris/c-auris-infection-control.html). These agents are active against Clostridium difficile spores and are used to clean surfaces contaminated with this difficult-to-eradicate bacteria. Because these agents are not typically used for hospital cleaning, it is critical to identify C. auris-contaminated surfaces in order to properly clean them and reduce the risk of transmission.

\section{Does $C$. auris Form Biofilms in Healthcare Settings?}

Candida spp. frequently form biofilms on medical surfaces, growing as adherent communities of cells encased in an extracellular matrix [29, 30]. Biofilms have been implicated in a variety of medical device infections, including urinary catheters, central venous catheters, cardiac-implanted devices, dentures, and other prostheses [21, 24]. Clinical studies of $C$. auris report high rates of catheters as the source of bloodstream infection, consistent with a role for biofilm in the pathogenesis of this organism [17]. Investigation of $C$. auris in a rodent model of catheter-associated bloodstream infection shows that isolates of this species adhere to catheter surfaces and proliferate as biofilms composed of yeast cells $\left[8^{\bullet}\right]$.

The capacity of $C$. auris to replicate as a biofilm extends to growth on skin, likely contributing to the organism's high propensity for skin colonization $[3,6,10,14]$. On porcine skin ex vivo, $C$. auris grows to a greater than 10 -fold burden when compared with $C$. albicans and replicates as an adherent community of multiple yeast layers [10]. C. auris also exhibits enhanced biofilm growth in synthetic sweat media in vitro, forming biofilms with burdens many fold greater than C. albicans. The characteristic of robust biofilm formation in skin milieu conditions presumably relates to the propensity of this organism to cause catheter-associated bloodstream infection. During implantation, catheter insertion through skin may serve port of entry for infection.

In addition to the role of biofilm formation for $C$. auris infection, this mode of growth likely plays a role in the persistence of $C$. auris in healthcare settings. Laboratory research studies have shown $C$. auris to survive on plastics and metals for up to 14 days, even in dry conditions $[13,28]$. Compared to $C$. albicans, $C$. auris biofilms formed in synthetic sweat media withstand longer periods of desiccation in the environment [10]. Thus, biofilm formation is a potential mechanism to understand how $C$. auris survives on medical equipment and hospital surfaces $[2,3,6]$.

\section{What Is the Influence of $C$. auris Biofilm Formation on Drug Resistance?}

For many Candida species, formation of a biofilm allows the cells to tolerate antifungals at concentrations many fold greater than those needed to kill their planktonic counterparts [31-36]. The degree of this biofilmassociated drug resistance varies by species and antifungal, with biofilms withstanding up to $1000 \times$-fold higher concentrations of antifungals compared to planktonic cells. Consequently, one would speculate that biofilm formation is likely to be associated with increased antifungal tolerance for $C$. auris as well. Indeed, C. auris biofilms exhibit increased resistance to antifungals from each of the available drug classes (Table 1) $[8 \bullet, 11 \bullet$, 37].

One of the largest concerns in the emergence of C. auris is this organism's frequent resistance to antifungals, which is observed even under planktonic conditions. Worldwide, nearly all isolates exhibit resistance to the triazole drug, fluconazole, and many (near $40 \%$ ) show a multidrug resistance phenotype $[2,6,11 \bullet \bullet, 38]$. Reports have also revealed pan-resistant isolates that display resistance to all three commonly prescribed drug classes [39]. The additional resistance associated with biofilm growth further complicates treatment. For example, echinocandin drugs are often used for treatment of invasive $C$. auris disease, as drug resistance is least frequent for this drug class $[2,40,41]$. However, given the $2-512 \times$ increase in resistance for biofilm, these drugs are not expected to be effective for treatment of $C$. auris infections involving biofilm growth. Similar to the other antifungal drug classes, the concentrations of echinocandin drugs needed to inhibit C. auris biofilms (MIC 90\% inhibition, Table 1) 
Table 1 Influence of biofilm formation on resistance to antifungal drugs

\begin{tabular}{|c|c|c|c|c|}
\hline Drug class & Anti-infective & 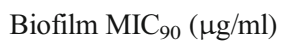 & Observation & Reference \\
\hline \multirow[t]{2}{*}{ Triazole } & Fluconazole & $>32$ & Resistance for planktonic and biofilm & {$[8 \bullet, 11 \bullet \bullet, 37]$} \\
\hline & Voriconazole & $>32$ & Biofilms 2 to $>32 \times$ more resistant & {$[11 \bullet \bullet, 37]$} \\
\hline \multirow[t]{2}{*}{ Polyene } & Amphotericin B deoxycholate & 2 to $>256$ & Biofilms 4 to $>512 \times$ more resistant & {$[11 \bullet \bullet, 37]$} \\
\hline & Liposomal amphotericin B & $2-16$ & Biofilms 4-32× more resistant & {$[11 \bullet \bullet]$} \\
\hline \multirow[t]{2}{*}{ Echinocandin } & Caspofungin & $>32$ & Biofilms $2-256 \times$ more resistant & {$[11,37]$} \\
\hline & Micafungin & 0.25 to $>32$ & Biofilms 4 to $>512 \times$ more resistant & {$[11 \bullet \bullet]$} \\
\hline
\end{tabular}

are above the levels that can safely be administered to patients.

The mechanism of resistance for $C$. auris biofilms appears to be multifactorial. Analysis of the extracellular matrix of C. auris biofilms reveals the presence of a mannan-glucan complex [8•]. These polysaccharides sequester antifungal drugs, preventing them from reaching their intracellular targets $[8 \cdot, 42,43]$. This antifungal sequestration has been shown to be involved in resistance to fluconazole for $C$. auris biofilms [8•]. However, drug sequestration may be involved in resistance to other antifungals as well. For C. albicans biofilms, extracellular matrix polysaccharides have been linked to a multidrug resistance mechanism, including resistance to amphotericin B, echinocandins, and flucytosine [44, 45].

Drug efflux pumps also appear to play a significant role in drug resistance for $C$. auris during biofilm growth [9•]. C. auris biofilm maturation involves an increasing abundance in transcripts encoding efflux pumps, including the major facilitator superfamily transporter MDRl and the ATP-binding cassette transporter $C D R 1$. These changes correlate with increased efflux pump activity and drug tolerance. Furthermore, disruption of efflux activity enhances the action of fluconazole against $C$. auris biofilms. A similar involvement of efflux pumps for Candida biofilm resistance has been described for C. albicans [46, 47]. However, in C. albicans, this mechanism primarily accounts for azole resistance during the very early stages of biofilm formation.

Further understanding of how biofilm formation by C. auris influences drug resistance is needed to develop new treatment strategies. For example, one study suggests that disruption of the quorum sensing pathways involved in fungal signaling can enhance the activity of echinocandin drugs [48]. Additionally, ibrexafungerp (SCY-078), an antifungal currently in clinical trials, exhibits activity against $C$. auris biofilms [7••]. This triterpenoid glycoside is the first drug in a new class of $\beta-1,3$ glucan synthesis inhibitors. Additional studies will be important to determine how these and other strategies targeting $C$. auris biofilm formation may be incorporated into treatment of $C$. auris infection.

\section{Conclusion}

Recent studies on globally emergent $C$. auris show how biofilm formation plays a major role in $C$. auris outbreaks in healthcare settings. $C$. auris exhibits a capacity to efficiently colonize skin, subsequently causing catheter-associated bloodstream infections and invasive candidiasis. Skin conditions promote high-burden biofilm formation which likely predisposes to catheter infections, environmental contamination, and spread among patients. Furthermore, biofilms formed on artificial surfaces tolerate high concentrations of antifungals, a serious problem regarding this pathogen that often displays multidrug resistance.

Future study will be critical for identifying triggers for C. auris biofilm formation and signaling pathways involved in this response to develop new therapeutic approaches. For example, it is unclear how the skin microbiome may influence C. auris growth, biofilm formation, and host responses. Understanding this process may shed light on strategies to derail colonization. In addition, the regulation of biofilm formation may vary significantly from $C$. albicans given the unique characteristics of $C$. auris. Delineating these pathways may provide potential novel drug targets Overall, expansion of our understanding of $C$. auris biofilm formation will be important to develop new tactics to control outbreaks and treat this devastating invasive disease.

Funding Information JEN is supported by the National Institutes of Health (R01 AI145939), the Burroughs Wellcome Fund (1012299), and the Doris Duke Charitable Foundation (2580130).

\section{Compliance with Ethical Standards}

Conflict of Interest The authors declare that they have no conflict of interest.

Human and Animal Rights and Informed Consent All reported studies/ experiments with human or animal subjects performed by the authors have been previously published and complied with all applicable ethical standards (including the Helsinki declaration and its amendments, institutional/national research committee standards, and international/national/institutional guidelines). 
Open Access This article is licensed under a Creative Commons Attribution 4.0 International License, which permits use, sharing, adaptation, distribution and reproduction in any medium or format, as long as you give appropriate credit to the original author(s) and the source, provide a link to the Creative Commons licence, and indicate if changes were made. The images or other third party material in this article are included in the article's Creative Commons licence, unless indicated otherwise in a credit line to the material. If material is not included in the article's Creative Commons licence and your intended use is not permitted by statutory regulation or exceeds the permitted use, you will need to obtain permission directly from the copyright holder. To view a copy of this licence, visit http://creativecommons.org/licenses/by/4.0/.

\section{References}

Papers of particular interest, published recently, have been highlighted as:

- Of importance

•- Of major importance

1. Satoh K, Makimura K, Hasumi Y, Nishiyama Y, Uchida K, Yamaguchi H. Candida auris sp nov., a novel ascomycetous yeast isolated from the external ear canal of an inpatient in a Japanese hospital. Microbiol Immunol. 2009;53(1):41-4. https://doi.org/10. 1111/j.1348-0421.2008.00083.x.

2. Lockhart SR, Etienne KA, Vallabhaneni S, Farooqi J, Chowdhary A, Govender NP, et al. Simultaneous emergence of multidrugresistant Candida auris on 3 continents confirmed by wholegenome sequencing and epidemiological analyses. Clin Infect Dis. 2017;64(2):134 40. https://doi.org/10.1093/cid/ciw691.

3. Schelenz S, Hagen F, Rhodes JL, Abdolrasouli A, Chowdhary A, Hall A, et al. First hospital outbreak of the globally emerging Candida auris in a European hospital. Antimicrob Resist Infect Control. 2016;5(35). https://doi.org/10.1186/s13756-016-0132-5.

4. Clancy CJ, Nguyen MH. Emergence of Candida auris: an international call to arms. Clin Infect Dis. 2017;64(2):141-3. https://doi. org/10.1093/cid/ciw696.

5. Lockhart SR, Berkow EL, Chow N, Welsh RM. Candida auris for the clinical microbiology laboratory: not your grandfather's Candida species. Clin Microbiol Newsl. 2017;39(13):99-103. https://doi.org/10.1016/j.clinmicnews.2017.06.003.

6. Adams E, Quinn M, Tsay S, Poirot E, Chaturvedi S, Southwick K, et al. Candida auris in healthcare facilities, New York, USA, 20132017. Emerg Infect Dis. 2018;24(10):1816-24. https://doi.org/10. 3201/eid2410.180649.

7••. . Larkin E, Hager C, Chandra J, Mukherjee PK, Retuerto M, Salem I, et al. The emerging pathogen candida auris: growth phenotype, virulence factors, activity of antifungals, and effect of SCY-078, a novel glucan synthesis inhibitor, on growth morphology and biofilm formation. Antimicrob Agents Chemother. 2017;61(5). https://doi.org/10.1128/AAC.02396-16 This study describes the adherence properties of $C$. auris and its capacity for biofilm formation.

8•. . Dominguez EG, Zarnowski R, Choy HL, Zhao M, Sanchez H, Nett JE, et al. Conserved role for biofilm matrix polysaccharides in Candida auris drug resistance. mSphere. 2019;4(1). https://doi.org/ 10.1128/mSphereDirect.00680-18 This work demonstrates that $C$. auris forms biofilms on vascular catheters in vivo and describes a mechanism for drug resistance during biofilm growth.
9•. Kean R, Delaney C, Sherry L, Borman A, Johnson EM, Richardson MD, et al. Transcriptome assembly and profiling of Candida auris reveals novel insights into biofilm-mediated resistance. mSphere. 2018;3(4). https://doi.org/10.1128/mSphere. 00334-18 This study shows temporal changes in the $\boldsymbol{C}$. auris transcriptome throughout the process of biofilm formation, highlighting how these changes could affect antifungal resistance.

10. Horton MV, Johnson CJ, Kernien JF, Patel TD, Lam BC, Cheong JZA, et al. Candida auris forms high-burden biofilms in skin niche conditions and on porcine skin. mSphere. 2020;5(1). https://doi. org/10.1128/mSphere.00910-19.

11•. . Sherry L, Ramage G, Kean R, Borman A, Johnson EM, Richardson MD, et al. Biofilm-forming capability of highly virulent, multidrug-resistant Candida auris. Emerg Infect Dis. 2017;23(2):328-31. https://doi.org/10.3201/eid2302.161320 This study shows that $C$. auris biofilms display increased tolerance of common antifungals when compared to planktonic yeast.

12. Calvo B, Melo ASA, Perozo-Mena A, Hernandez M, Francisco EC, Hagen F, et al. First report of Candida auris in America: clinical and microbiological aspects of 18 episodes of candidemia. J Inf Secur. 2016;73(4):369-74. https://doi.org/10.1016/j.jinf.2016.07.008.

13. Sayeed MA, Farooqi J, Jabeen K, Awan S, Mahmood SF. Clinical spectrum and factors impacting outcome of Candida auris: a single center study from Pakistan. BMC Infect Dis. 2019;19(384). https:// doi.org/10.1186/s12879-019-3999-y.

14. Escandón P, Chow NA, Caceres DH, Gade L, Berkow EL, Armstrong P, et al. Molecular epidemiology of Candida auris in Colombia reveals a highly related, countrywide colonization with regional patterns in amphotericin B resistance. Clin Infect Dis. 2018;68:15-21. https://doi.org/10.1093/cid/ciy411.

15. Rudramurthy SM, Chakrabarti A, Paul RA, Sood P, Kaur H, Capoor MR, et al. Candida auris candidaemia in Indian ICUs: analysis of risk factors. J Antimicrob Chemother. 2017;72(6):1794 801. https://doi.org/10.1093/jac/dkx034.

16. Garcia-Bustos V, Salavert M, Ruiz-Gaitan AC, Cabanero-Navalon MD, Sigona-Giangreco IA, Peman J. A clinical predictive model of candidaemia by Candida auris in previously colonized critically ill patients. Clin Microbiol Infect. 2020. https://doi.org/10.1016/j.cmi. 2020.02.001.

17. Sayeed MA, Farooqi J, Jabeen K, Mahmood SF. Comparison of risk factors and outcomes of Candida auris candidemia with nonCandida auris candidemia: a retrospective study from Pakistan. Med Mycol. 2019. https://doi.org/10.1093/mmy/myz112.

18. Akrabarti A. Candida auris candidaemia in an intensive care unit prospective observational study to evaluate epidemiology, risk factors, and outcome. J Crit Care. 2020;57:42-8. https://doi.org/10. 1016/j.jcrc.2020.01.004.

19. van Schalkwyk E, Ruth SM, Juno T, Liliwe S, Husna I, Warren L, et al. Epidemiologic shift in Candidemia driven by Candida auris, South Africa, 2016-2017. Emerg Infect Dis. 2019;25(9):1698-707. https://doi.org/10.3201/eid2509.190040.

20. Park JY, Bradley N, Brooks S, Burney S, Wassner C. Management of Patients with Candida auris Fungemia at community hospital, Brooklyn, New York, USA, 2016-2018. Emerg Infect Dis. 2019;25(3):601-2. https://doi.org/10.3201/eid2503.180927.

21. Ramage G, Martínez JP, López-Ribot JL. Candida biofilms on implanted biomaterials: a clinically significant problem. FEMS Yeast Res. 2006;6:979-86. https://doi.org/10.1111/j.1567-1364.2006. 00117.x.

22. Khatamzas E, Madder H, Jeffery K. Neurosurgical deviceassociated infections due to Candida auris - three cases from a single tertiary center. J Inf. 2019;78(5):409-10. https://doi.org/10. 1016/j.jinf.2019.02.004. 
23. Roberts SC, Zembower TR, Bolon MK, Kadakia AR, Gilley JH, $\mathrm{Ko} \mathrm{JH}$, et al. Successful treatment of a Candida auris intra-articular infection. Emerg Microbes Infect. 2019;8:866-8. https://doi.org/10. 1080/22221751.2019.1625287.

24. Kojic EM, Darouiche RO. Candida infections of medical devices. Clin Microbiol Rev. 2004;17(2):255-67.

25. Eyre DW, Sheppard AE, Madder H, Moir I, Moroney R, Quan TP, et al. A Candida auris outbreak and its control in an intensive care setting. N Engl J Med. 2018;379(14):1322-31. https://doi.org/10. 1056/NEJMoa1714373.

26. Biswal M, Rudramurthy SM, Jain N, Shamanth AS, Sharma D, Jain $\mathrm{K}$, et al. Controlling a possible outbreak of Candida auris infection: lessons learnt from multiple interventions. J Hosp Infect. 2017;97: 363-70. https://doi.org/10.1016/j.jhin.2017.09.009.

27. Welsh RM, Bentz ML, Shams A, Houston H, Lyons A, Rose LJ, et al. Survival, persistence, and isolation of the emerging multidrugresistant pathogenic yeast Candida auris on a plastic health care surface. J Clin Microbiol. 2017;55(10):2996-3005. https://doi.org/ 10.1128/JCM.00921-17.

28. Cadnum J, Shaikh A, Piedrahita C, Sankar T, Jencson A, Larkin E, et al. Effectiveness of disinfectants against Candida auris and other Candida species. Infect Control Hosp Epidemiol. 2017;38(10): 1240-3.

29. Donlan RM. Biofilm formation: a clinically relevant microbiological process. Clin Infect Dis. 2001;33(8):1387-92.

30. Mukherjee PK, Zhou G, Munyon R, Ghannoum MA. Candida biofilm: a well-designed protected environment. Med Mycol. 2005;43(3):191-208. https://doi.org/10.1080/ 13693780500107554 .

31. Mukherjee PK, Chandra J, Kuhn DM, Ghannoum MA. Mechanism of fluconazole resistance in Candida albicans biofilms: phasespecific role of efflux pumps and membrane sterols. Infect Immun. 2003;71(8):4333-40.

32. Kuhn DM, Chandra J, Mukherjee PK, Ghannoum MA. Comparison of biofilms formed by Candida albicans and Candida parapsilosis on bioprosthetic surfaces. Infect Immun. 2002;70(2):878-88.

33. Ramage G, Vandewalle K, Wickes BL, Lopez-Ribot JL. Characteristics of biofilm formation by Candida albicans. Rev Iberoam Micol. 2001;18(4):163-70.

34. Chandra J, Mukherjee PK, Leidich SD, Faddoul FF, Hoyer LL, Douglas LJ, et al. Antifungal resistance of candidal biofilms formed on denture acrylic in vitro. J Dent Res. 2001;80(3):903-8.

35. Baillie GSLJD. Matrix polymers of Candida biofilms and their possible resistance to antifungal agents. J Antimicrob Chemother. 2000;46:397-403.

36. Hawser SP, Douglas LJ. Resistance of Candida albicans biofilms to antifungal agents in vitro. Antimicrob Agents Chemother. 1995;39(9):2128-31.

37. Romera D, Aguilera-Correa JJ, Gadea I, Vinuela-Sandoval L, Garcia-Rodriguez J, Esteban J. Candida auris: a comparison between planktonic and biofilm susceptibility to antifungal drugs. $\mathrm{J}$ Med Microbiol. 2019;68(9):1353-8. https://doi.org/10.1099/jmm. 0.001036 .
38. Chowdhary A, Sharma C, Meis JF. Candida auris: a rapidly emerging cause of hospital-acquired multidrug-resistant fungal infections globally. PLoS Pathog. 2017;13(5):e1006290-e. https://doi.org/10. 1371/journal.ppat.1006290.

39. Ostrowsky B, Greenko J, Adams E, Quinn M, O'Brien B, Chaturvedi V, et al. Candida auris isolates resistant to three classes of antifungal medications - New York, 2019. MMWR Morb Mortal Wkly Rep. 2020;69(1):6-9. https://doi.org/10.15585/ mmwr.mm6901a2.

40. Chowdhary A, Prakash A, Sharma C, Kordalewska M, Kumar A, Sarma S, et al. A multicentre study of antifungal susceptibility patterns among 350 Candida auris isolates (2009-17) in India: role of the ERG11 and FKS1 genes in azole and echinocandin resistance. J Antimicrob Chemother. 2018;73:891-9. https://doi.org/10.1093/ $\mathrm{jac} / \mathrm{dkx} 480$.

41. Pfaller MA, Diekema DJ, Turnidge JD, Castanheira M, Jones RN. Twenty years of the SENTRY antifungal surveillance program: results for Candida species from 1997-2016. Open Forum Infect Dis. 2019;6(1):S79-94. https://doi.org/10.1093/ofid/ofy358.

42. Mitchell KF, Taff HT, Cuevas MA, Reinicke EL, Sanchez H, Andes DR. Role of matrix $\beta-1,3$ glucan in antifungal resistance of non-albicans Candida biofilms. Antimicrob Agents Chemother. 2013;57(4):1918-20. https://doi.org/10.1128/AAC.02378-12.

43. Mitchell KF, Zarnowski R, Sanchez H, Edward JA, Reinicke EL, Nett JE, et al. Community participation in biofilm matrix assembly and function. Proc Natl Acad Sci U S A. 2015;112(13):4092-7. https://doi.org/10.1073/pnas.1421437112.

44. Nett JE, Crawford K, Marchillo K, Andes DR. Role of Fks1p and matrix glucan in Candida albicans biofilm resistance to an echinocandin, pyrimidine, and polyene. Antimicrob Agents Chemother. 2010;54(8):3505-8. https://doi.org/10.1128/AAC. 00227-10.

45. Vediyappan G, Rossignol T, d'Enfert C. Interaction of Candida albicans biofilms with antifungals: transcriptional response and binding of antifungals to beta-glucans. Antimicrob Agents Chemother. 2010;54(5):2096-111. https://doi.org/10.1128/AAC. 01638-09.

46. Mukherjee PK, Chandra J, Kuhn DM, Ghannoum MA. Mechanism of fluconazole resistance in Candida albicans biofilms: phasespecific role of efflux pumps and membrane sterols. Infect Immun. 2003;71(8):4333-40. https://doi.org/10.1128/iai.71.8. 4333-4340.2003.

47. Ramage G, Bachmann S, Patterson TF, Wickes BL, López-Ribot JL. Investigation of multidrug efflux pumps in relation to fluconazole resistance in Candida albicans biofilms. J Antimicrob Chemother. 2002;49(6):973-80. https://doi.org/10.1093/jac/ dkf049.

48. Nagy F, Toth Z, Daroczi L, Szekely A, Borman AM, Majoros L, et al. Farnesol increases the activity of echinocandins against Candida auris biofilms. Med Mycol. 2019;58:404-7. https://doi. org/10.1093/mmy/myz057.

Publisher's Note Springer Nature remains neutral with regard to jurisdictional claims in published maps and institutional affiliations. 